\title{
Les eaux minérales sulfurées Problématique générale
}

\author{
Mme C. Ohayon-Courtès \\ Université de Bordeaux 2
}

\section{GÉNÉRALITÉS}

\subsection{Définition}

Les eaux minérales sulfurées se distinguent par leurs propriétés organoleptiques particulières :

- en premier lieu, par leur odeur dite d'œuf pourri, c'est-à-dire présentant l'odeur de l'hydrogène sulfuré $\left(\mathrm{H}_{2} \mathrm{~S}\right)$,

- puis par leur instabilité au contact de l'air qui, dans certains cas, fait apparaître une coloration jaune voire brune ou une opalescence blanche.

$\mathrm{Ce}$ sont ces caractéristiques qui les ont faites distinguer depuis fort longtemps $[1,8]$ sous le terme inadapté d'eau sulfureuse. Une abondante bibliographie témoigne de l'intérêt suscité par ces eaux [4].

\subsection{Classification [6]}

C"est sur la base des caractères organoleptiques cités que la classe des Eaux Sulfurées a été créée, sans préjuger de l'aspect quantitatif puisqu'on y trouve des eaux à $1 \mathrm{mg} / \mathrm{l}$ comme à $250 \mathrm{mg} / \mathrm{l}$ de sulfures.

Ultérieurement, deux subdivisions sont apparues en fonction du cation dominant :
- les eaux sulfurées sodiques,

- les eaux sulfurées calciques.

Ces appellations ont parfois été contestées [9] mais sont toujours largement admises, il faut peut-être les considérer comme purement conventionnelles. Quoi qu'il en soit, cette distinction sur le cation dominant permet de ranger les eaux sulfurées dans deux groupes assez homogènes d'eaux fondamentalement différentes (tabl. l).

$\mathrm{La}$ définition précédente, toujours en vigueur aujourd'hui, et qui consiste à ne considérer que l'odeur caractéristique semble insuffisante :

- au plan quantitatif : l'odorat est le mode de détection le plus fin de I'hydrogène sulfuré, autrement dit pourraient être classées dans ce groupe des eaux dont la concentration en sulfures serait inférieure à $0,1 \mathrm{mg} / \mathrm{l}$.

- au plan physico-chimique: I'odeur particulière n'est pas l'apanage exclusif de l'hydrogène sulfuré, mais aussi d'autres dérivés du soufre de degré redox $(-2)$, comme les polysulfures ou les sulfures organiques.

— au plan de l'origine géologique : si le groupe des eaux sulfurées sodiques est homogène, celui des sulfurées calciques est assez disparate.

Enfin, cette définition prise au sens strict pourrait permettre d'englober des eaux qui ne répondent ensuite à aucun des deux sous-groupes, mais qui possèdent un caractère réducteur à l'émergence comme certaines eaux

\section{Sulphurous mineral waters \\ The general problem}

\begin{abstract}
Sulphurous mineral waters have been known for a very long time, but their geological origin and classification are still the subject of debate.

From the standpoint of physical chemistry, thermodynamics provides a large number of data most of which cannot be checked experimentally because the analysis of these waters represents a major problem for the chemist despite the development of special techniques.

Physical chemical investigation is complicated by the fact that these waters are unstable in contact with the air. This phenomenon is of particular importance because the reduced sulphur species that appear after emergence of the water will be more or less suitable for pharmacological use although their appearance will depend in particular on the operating conditions.
\end{abstract}


Tableau 1. - Caractéristiques principales des deux classes d'eaux minérales sulfurées.

\begin{tabular}{|c|c|c|}
\hline Critères & Eaux sulfurées sodiques & Eaux sulfurées calciques \\
\hline Température & hyperthermale jusqu'à $72^{\circ} \mathrm{C}$ & le plus souvent froides \\
\hline Minéralisation totale & faible & le plus souvent supérieure à $1 \mathrm{~g} / \mathrm{l}$ \\
\hline $\mathrm{pH}$ & de 8 à 10 & de 6,5 à 7,5 \\
\hline Cation majeur & $\begin{array}{c}\text { Sodium : } 90 \text { à } 95 \% \text { de la totalité } \\
\text { des cations }\end{array}$ & Calcium \\
\hline \multirow[t]{2}{*}{ Autres caractéristiques } & $\begin{array}{c}\text { Magnésium : à l'état de traces }\left(10^{-6} \mathrm{M} / \mathrm{l}\right) \\
\text { marqueur de mélange }\end{array}$ & \\
\hline & $\begin{array}{l}\text { Silice : jusqu'à } 50 \% \text { de la } \\
\text { minéralisation totale }\end{array}$ & \\
\hline Origine géologique & $\begin{array}{c}\text { terrains cristallins, granite } \\
\text { pyrite }\end{array}$ & $\begin{array}{l}\text { terrains divers, plutôt sédimentaires } \\
\text { réduction chimique et/ou biologique } \\
\text { du sulfate }\end{array}$ \\
\hline Localisation principale & Chaîne pyrénéenne et Corse & diffuse (piémonts) \\
\hline
\end{tabular}

ferrugineuses riches en sulfate et qui contiennent de façon très transitoire du soufre réduit.

\subsection{Répartition géographique [4]}

Les eaux minérales sulfurées constituent une classe d'eaux largement représentée puisque sur 105 villes thermales françaises, près de 40 en exploitent au moins une (fig. I).

\subsection{Indications thérapeutiques}

Elles sont :

- en premier lieu, les affections O.R.L., en nébulisations, aérosols, humages, douches pharyngiennes, etc... Certains établissements utilisent aussi les gaz recueillis à l'émergence ou extraits (pour les insufflations) et les vapeurs d'eau,

- mais aussi, la rhumatologie et les séquelles de traumatismes ostéo-articulaires, en bains, massages, douches, etc... Cette indication est devenue aujourd'hui aussi importante que la précédente compte tenu du nombre de curistes concernés,

- enfin, trois autres orientations thérapeutiques sont le fait de quelques stations : la dermatologie, la gynécologie et les affections des reins et des voies urinaires.

\section{II $\square$ ASPECTS PHYSICO-CHIMIQUES}

\subsection{Les espèces chimiques du soufre [11]}

Parmi la quarantaine d'espèces inorganiques connues du soufre, les plus communes et les plus stables se retrouvent dans les eaux minérales sulfurées ainsi que des espèces organiques, beaucoup plus mal connues (tabl. 2).

Par définition une eau sulfurée contient du soufre de degré $(-2)$ mais la thermodynamique montre que toutes ces espèces peuvent coexister théoriquement puisqu'elles sont reliées par des réactions d'équilibre :
- entre espèces d'un même degré redox par des réactions d'équilibre $\mathrm{pH}$;

- entre deux espèces de degrés redox différents par des réactions d'oxydoréduction ;

- entre trois espèces différentes on a montré aussi que des réactions de dismutation étaient possibles dont la principale concerne la formation de polysulfures à partir de $\mathrm{S}(-2)$ et de $\mathrm{S}(0)$ lorsqu'ils coexistent.

BOULÈGUe [2] a montré que ces équilibres pouvaient être reproduits expérimentalement en solution et que les eaux sulfurées naturelles répondent à ces systèmes théoriques.

Les eaux minérales sulfurées sont donc des systèmes complexes et toute modification des paramètres de ce système (pression, température, gaz dissous et $\mathrm{pH}$ ) perturbera la répartition des espèces du soufre.

La connaissance des eaux sulfurées passe donc non seulement par le dosage des espèces présentes mais aussi par l'étude des équilibres mis en jeu.

\subsection{L'analyse des eaux sulfurées}

Pour établir la composition en dérivés du soufre d'une eau sulfurée, il faudra donc établir la répartition des différentes espèces. Cela met en jeu trois procédés.

\subsubsection{Les méthodes de dosage}

Malheureusement, il n'existe pas de méthode de dosage parfaitement spécifique d'une espèce du soufre, ce qui se conçoit aisément du fait des propriétés chimiques très voisines de ces dérivés et de l'incidence des uns sur les autres.

La méthode la plus ancienne et la plus communément employée est la iodométrie qui met à profit les propriétés réductrices de ces composés. Elle est encore utilisée mais conduit à des résultats insuffisamment fins et demande de lourdes manipulations qui inévitablement perturbent l'échantillon.

La méthode de référence est la méthode potentiométrique de BOULÈGUE et POPOFF [3] avec une électrode sé- 


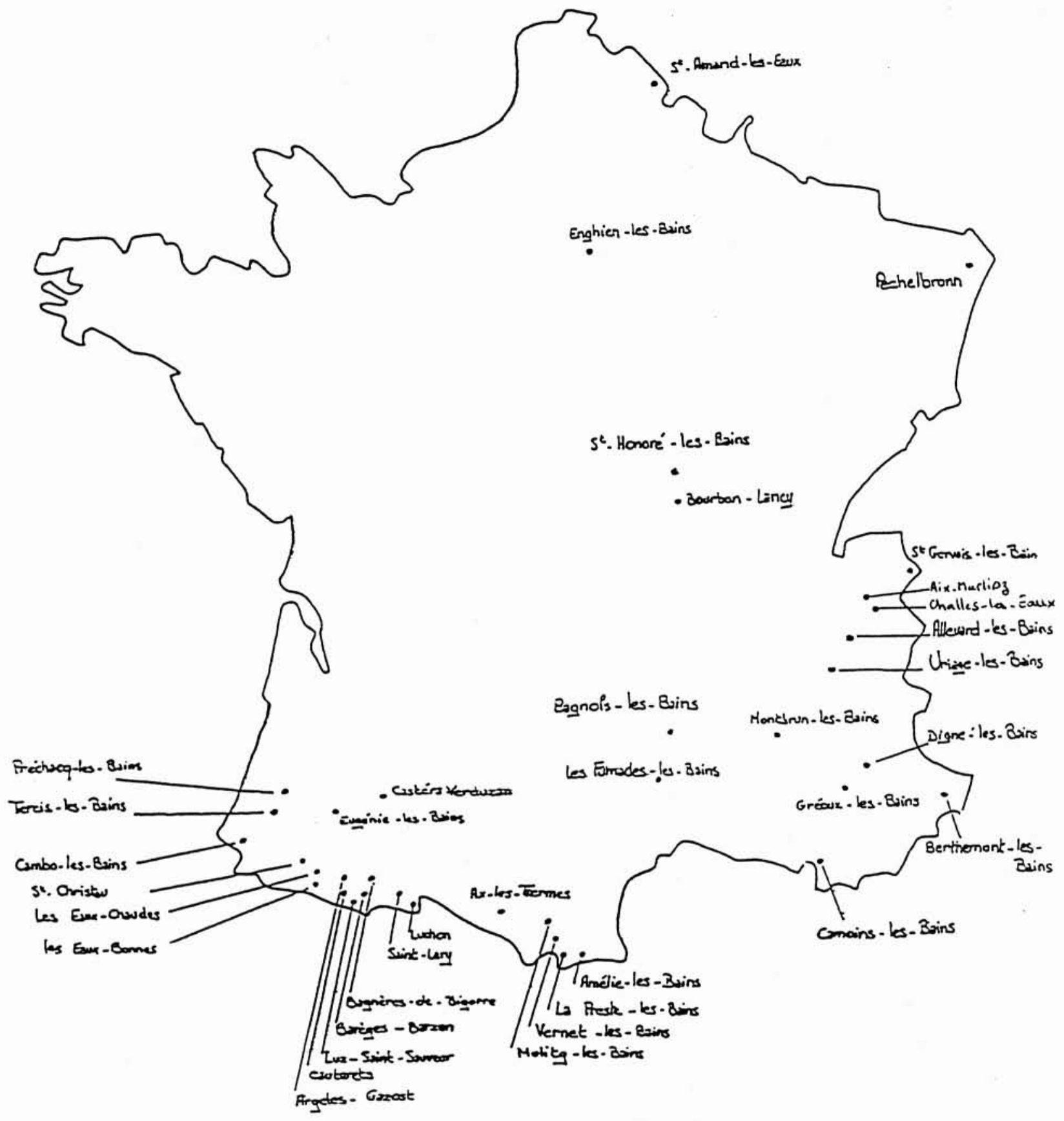

1. Principales stations thermales exploitant des eaux sulfurées en France métropolitaine.

lective du $\mathrm{S}(-2)$ ionisable (qu'il soit minéral ou organique). Cette méthode conduit à :

$\Sigma\left[\mathrm{H}_{2} \mathrm{~S}\right],\left[\mathrm{S}_{2} \mathrm{O}_{3}--\right]$ avec précision et un seuil de détection faible ;

$\Sigma\left[\mathrm{HSO}_{3}-\right]$ avec une précision et un seuil de détection variables ;

$\Sigma\left[\mathrm{HS}_{\mathrm{n}} \mathrm{S}--\right]$ avec une précision et un seuil de détection moyens :

le $\mathrm{S}(-2)$ organique est caractérisé mais sa quantification est aléatoire, voire impossible, et en tout cas toujours arbitraire.
Cette méthode est beaucoup plus commode que la précédente mais demande une certaine expérience et un matériel plus sophistiqué. Bien qu'elle représente un formidable progrès, elle n'est pas suffisante et doit être couplée à d'autres déterminations comme celles du soufre total $[S]_{T}$, du soufre élémentaire $[S(0)]$ et du sulfate $[S(+6)]$.

De nouvelles méthodes instrumentales comme la chromatographie ionique ou la chromatographie gazeuse couplée à la spectrométrie de masse ou la spectrométrie de masse couplée à l'ICP devraient permettre d'approfondir 
Tableau 2. - Les espèces du soufre classées par degré redox et présentes en solution aqueuse.

\begin{tabular}{|c|c|c|c|c|}
\hline & Degré redox & Espèces chimiques & Appellations courantes & pKa \\
\hline \multirow{5}{*}{ Etats réduits } & $(-2)$ & $\begin{array}{l}\mathrm{H}_{2} \mathrm{~S} \text { : hydrogène sulfuré } \\
\mathrm{HS}^{-}: \text {sulfhydrate } \\
\left(\mathrm{S}^{--}: \text {sulfure }\right)\end{array}$ & $\Sigma\left[\mathrm{H}_{2} \mathrm{~S}\right]$ sulfures & $\begin{array}{r}7,01 \\
14\end{array}$ \\
\hline & $-1<d<0$ & $\begin{array}{l}\left(\mathrm{H}_{2} \mathrm{~S}_{\mathrm{n}} \mathrm{S} \text { : polysulfanes) }\right. \\
\mathrm{HS}_{\mathrm{n}} \mathrm{S}^{-} \text {: polysulfhydrates } \\
\mathrm{S}_{\mathrm{n}} \mathrm{S}^{--} \text {polysulfures } \\
1<\mathrm{n}<4\end{array}$ & $\begin{array}{l}\Sigma\left[\mathrm{HS}_{m}\right] \text { polysulfures } \\
2<m<5\end{array}$ & $\begin{array}{l}<4 \\
<6,5\end{array}$ \\
\hline & 0 & $\begin{array}{l}\mathrm{S}_{0}: \text { soufre colloïdal } \\
\mathrm{S}_{8}: \text { soufre rhomboédrique }\end{array}$ & $\mathrm{S}(0)$ soufre élémentaire & \\
\hline & +2 & $\mathrm{~S}_{2} \mathrm{O}_{3}{ }^{--}$: thiosulfate & & \\
\hline & +4 & $\begin{array}{l}\left(\mathrm{H}_{2} \mathrm{SO}_{3}: \text { acide sulfureux }\right) \\
\mathrm{HSO}_{3}^{-}: \text {hydrogénosulfite } \\
\mathrm{SO}_{3}{ }^{--} \text {sulfite }\end{array}$ & $\Sigma\left[\mathrm{H}_{2} \mathrm{SO}_{3}\right]$ sulfites & $\begin{array}{l}1,77 \\
7,2\end{array}$ \\
\hline Oxydé & +6 & $\begin{array}{l}\left(\mathrm{H}_{2} \mathrm{SO}_{4}: \text { acide sulfurique) }\right. \\
\text { ( } \mathrm{HSO}_{4}^{-}: \text {hydrogénosulfate) } \\
\mathrm{SO}_{4}^{-}: \text {sulfate }\end{array}$ & {$\left[\mathrm{SO}_{4}^{-}\right]$sulfate } & $\begin{array}{l}<1 \\
1,92\end{array}$ \\
\hline Organique & -2 & $\begin{array}{l}R-S H \\
R-S-R^{\prime} \\
R-S-S-R^{\prime} \\
R-S_{n}-R^{\prime}\end{array}$ & $\begin{array}{l}\text { thiols } \\
\text { sulfures organiques } \\
\text { disulfures } \\
\text { polysulfures organiques }\end{array}$ & \\
\hline
\end{tabular}

Les espèces figurant entre parenthèses ne peuvent pas exister dans les eaux minérales naturelles en raison du pH.

nos connaissances des espèces du soufre mais exclusivement au laboratoire.

\subsubsection{Le calcul}

En fonction du $\mathrm{pH}$, de la température de l'eau et des pKa des différentes espèces, il est possible de calculer la répartition des formes d'un même degré redox.

\subsubsection{Le comportement électrochimique [2]}

Pour vérifier la validité de l'analyse, qui peut toujours être mise en cause par la simple manipulation de l'échantillon, il est possible d'étudier le comportement électrochimique de l'échantillon et le comparer aux diagrammes théoriques des systèmes thermodynamiques du soufre. Cela se réalise par la mesure des potentiels $E_{h}$ (fournis par une électrode de platine) et des potentiels $E_{S}$ (fournis par l'électrode spécifique) en faisant varier le $\mathrm{pH}$ mais en conservant tous les autres paramètres constants. On pourra ainsi vérifier que l'eau analysée répond à un système $\mathrm{H}_{2} \mathrm{~S}-\mathrm{H}_{2} \mathrm{O}$, dans lequel il est peu probable qu'apparaissent des polysulfures sans une étape d'oxydation préalable, ou à un système $\mathrm{H}_{2} \mathrm{~S}-\mathrm{S}_{8}-\mathrm{H}_{2} \mathrm{O}$ dont la stabilité passe par la formation de polysulfures.

\subsection{Paramètres influençant l'analyse}

Comme nous l'avons vu, une eau sulfurée est un système complexe qui aura tendance à évoluer à la moindre va- riation d'un paramètre du système. Certains ont une influence prépondérante :

\subsubsection{La température}

La température modifie les données des équilibres thermodynamiques et par voie de conséquence la répartition et la stabilité de certaines espèces. Sachant que les eaux minérales sulfurées sont souvent chaudes, sitôt l'émergence, les équilibres vont être modifiés et l'analyse ne rendra pas forcément compte de l'eau telle qu'elle est dans la nappe ou dans la colonne d'exore.

\subsubsection{La pression partielle en oxygène}

Initialement elle est extrêmement faible, voire inexistante, dans les eaux sulfurées, mais dès l'émergence elle se trouve modifiée par le contact à l'air. L'oxygène va favoriser l'oxydation des espèces les plus réduites.

\subsubsection{La pression partielle en anhydride carbonique}

Elle aussi se trouve modifiée dès le contact avec l'atmosphère, entraînant une variation du pH de l'eau lié à l'équilibre de l'acide carbonique. En conséquence, les équilibres $\mathrm{pH}$ et les réactions d'oxydoréduction ou de dismutation 
dépendantes du $\mathrm{pH}$ vont être déplacés et la répartition des dérivés du soufre sera perturbée.

\subsubsection{La pression partielle en hydrogène sulfuré}

La différence de concentration en $\mathrm{H}_{2} \mathrm{~S}$ de l'eau et de l'atmosphère est le plus souvent en faveur d'un dégazage de ce gaz, ce qui encore une fois modifie la répartition des différentes espèces du $\mathrm{S}(-2)$.

\subsection{Problématique}

Ainsi le problème majeur des eaux sulfurées réside dans l'établissement de leur composition chimique précise.

La notion d'analyse telle qu'à l'émergence prend ici tout son sens : il faut la réaliser sur place et le plus près possible du point d'émergence, ce qui techniquement n'est pas toujours réaliste.

Par ailleurs, les thermalistes s'intéressent plus à la qualité de l'eau telle qu'on l'utilise et non plus telle qu'à l'émergence, l'analyse doit donc aussi être établie aux points d'utilisation dans l'établissement de soins, ce qui conduit à des résultats variables en fonction de la technique d'exploitation.

Quoi qu'il en soit, l'analyse devra toujours être réalisée sur place, avec un maximum de précautions (travail à température constante et à l'abri de l'air). Elle ne sera de toute façon représentative que d'un instant donné, d'où la difficulté de la reproduire sans fluctuations parfois considérables.

Les fluctuations observées ne seront pas forcément à rapprocher de modifications géologiques ou hydrologiques ou géochimiques ou géodynamiques, mais aussi analytiques, ce qui explique les difficultés d'interpréter une analyse, de poser des hypothèses sur l'origine géologique et de se conformer à la législation des eaux minérales qui insiste sur la notion de constance de composition de l'eau.

Au plan de la surveillance réglementaire des eaux minérales dans les établissements thermaux, les eaux sulfurées constituent un réel problème pour les laboratoires. En effet, les textes prévoient que le contrôle périodique porte, entre autres paramètres, sur un anion caractéristique de l'eau minérale et il a été vivement souhaité que ce soit la sulfuration. Mais quelle espèce du soufre choisir : toutes ou seulement $\mathrm{H}_{2} \mathrm{~S}$ ? Utiliser un autre marqueur peut ne pas suffire, surtout si l'activité pharmacologique de ces eaux est uniquement rattachée aux dérivés du soufre.

En plus d'être réalisée sur place, l'analyse doit suivre un protocole extrêmement précis pour que les résultats, même s'ils ne sont pas justes en valeur absolue soient parfaitement comparables pour un même laboratoire mais aussi entre des laboratoires différents. De plus, multiplier les méthodes et les déterminations implique un matériel lourd et contraignant, un temps d'analyse plus long et un coût plus élevé pour l'exploitant.

Reste le problème des dérivés organiques du soufre. Au plan fondamental, il serait utile d'améliorer nos connaissances dans ce domaine puisqu'ils sont très liés à l'origine géologique de l'eau, à la possibilité d'infiltration d'eaux superficielles ou à la présence d'une flore microbienne particulière.

\section{III ÉVOLUTION \\ AU CONTACT \\ DE L'AIR -}

\subsection{Aspect théorique}

Comme nous l'avons déjà signalé à propos de l'analyse des eaux sulfurées, le contact avec l'atmosphère (en particulier l'effet des pressions partielles en oxygène et en hydrogène sulfuré) provoque des modifications des espèces chimiques initialement présentes dont certaines sont irréversibles.

L'oxydation conduirait théoriquement à la disparition des espèces les plus réduites au profit des espèces de degrés redox intermédiaires, pour finalement atteindre l'état le plus oxydé :

$$
\mathrm{S}(-2) \rightarrow \mathrm{S}(0) \rightarrow \mathrm{S}(+2) \rightarrow \mathrm{S}(+4) \rightarrow \mathrm{S}(+6)
$$

Ce schéma traditionnel peut être produit expérimentalement sur des solutions étalon de sulfure, mais le plus souvent les phénomènes d'oxydation sont beaucoup plus complexes.

\subsection{Etude expérimentale}

Les données expérimentales du comportement des eaux sulfurées au contact de l'air dont nous disposons concernent essentiellement les eaux sulfurées sodiques $[7,11]$ et quelques eaux sulfurées calciques [10].

Les phénomènes prépondérants observés sont:

- une augmentation transitoire de $\mathrm{pH}$, sur environ $1 \mathrm{~h}$ qui représente le temps pour atteindre l'équilibre thermique avec l'atmosphère ;

- une première étape d'oxydation du $\mathrm{S}(-2)$ en $\mathrm{S}(0)$ qui a lieu dès la première heure ;

- un dégazage très important de $\mathrm{H}_{2} \mathrm{~S}$ qui porte en moyenne sur $70 \%$ de la valeur initiale, pendant huit heures.

Ainsi, seulement $30 \%$ du soufre réduit subit les transformations oxydatives en solution.

Le schéma réactionnel de l'oxydation passe toujours par l'étape du soufre élémentaire mais peut ensuite suivre deux voies essentielles (fig. 2):

- une voie proche du schéma théorique : précipitation du $\mathrm{S}(0)$, apparition de thiosulfate, éventuellement de sulfite (il y a controverse sur cette espèce intermédiaire du fait de l'imprécision des techniques d'analyse) pour enfin donner du sulfate. L'eau de Luchon suit principalement ce modèle, - une deuxième voie, plus généralement observée, conduit à l'apparition de polysulfures par solubilisation du soufre élémentaire, augmentation des thiols, mais l'apparition des oxyanions n'est pas quantifiable.

Cette dernière voie correspond à une stabilisation du pouvoir réducteur de l'eau sur une période parfois très longue ( $72 \mathrm{~h})$.

Il est difficile d'établir a priori le sens de l'évolution d'une eau sulfurée mais en général il a été observé qu'une diffusion lente de l'oxygène de l'air (ratio $\Sigma\left[\mathrm{H}_{2} \mathrm{~S}\right] /\left[\mathrm{O}_{2}\right]$ élevé) favorise la voie des polysulfures. 


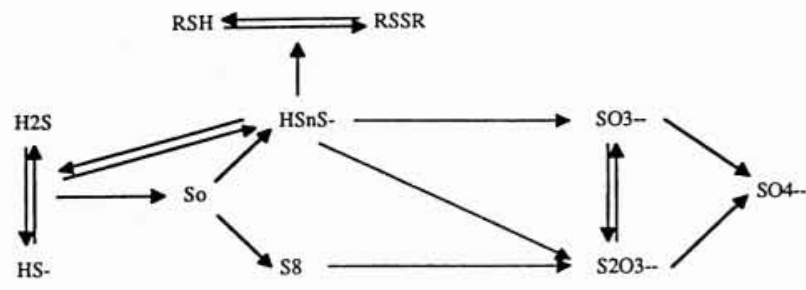

2. Schéma de l'oxydation des dérivés du soufre dans les eaux minérales sulfurées.

\subsection{Conséquences pratiques}

L'eau sulfurée en cours d'exploitation dans un établissement thermal n'aura pas la même composition en différents points du réseau ou aux différents postes de soins.

L'utilisation de l'eau en provenance directe du forage, sans échangeur thermique et sans réservoir tampon, limite l'évolution de l'eau qui a toutes les chances de rester « telle qu'à l'émergence » en amont des postes de soins.

S'il y a stockage, l'eau subira le schéma d'oxydation que nous avons établi précédemment mais à des degrés différents selon que le réservoir limite ou non le dégazage de $\mathrm{H}_{2} \mathrm{~S}$ (stockage à l'abri de l'air) et maintient ou non une pression partielle en $\mathrm{H}_{2} \mathrm{~S}$ élevée au-dessus de l'eau (stockage avec confinement de l'atmosphère). Limiter le dégazage favorisera dans tous les cas l'apparition de polysulfures et de thiols selon la deuxième voie d'oxydation de la figure 2.

Certains soins, comme les bains bouillonnants, favorisent la précipitation du $\mathrm{S}(0)$ et on assiste au blanchiment bien connu de l'eau dans les bains ou les piscines. Ce type de soins pose donc le problème de la conservation des qualités initiales de l'eau et ne se justifie que si l'eau est renouvelée au moment du soin pour chaque curiste en créant un «aérosol » de $\mathrm{H}_{2} \mathrm{~S}$.

La question qui reste posée est la suivante : les polysulfures, les thiols et les dérivés intermédiaires d'oxydation ont-ils une valeur pharmacologique qui pourrait justifier que leur apparition soit favorable?

\section{IV $\square$ ASPECT PHARMACOLOGIQUE}

Il est admis que la principale espèce active des eaux sulfurées est $\mathrm{H}_{2} \mathrm{~S}$ (ou $\mathrm{HS}^{-}$), ainsi, il a souvent été dit (et la réglementation s'y emploie), qu'il fallait conserver à l'eau ses qualités telles qu'à l'émergence.

Toutefois, quelques tests pharmacologiques ont montré qu'après oxydation partielle l'eau sulfurée restait active sur organes isolés et vis-à-vis de l'activité de divers médiateurs chimiques comme l'acétylcholine ou l'histamine [5, 11].

Ces études laissent supposer que les polysulfures et les thiols ne sont pas dénués d'activité et il est possible d'envisager que les modifications de l'eau qui favorisent l'apparition de ces dérivés soient, sinon souhaitables, tout au moins non rédhibitoires.

\section{ASPECT MICROBIOLOGIQUE}

Cet aspect doit être considéré sous deux angles différents : - au plan sanitaire : les eaux sulfurées sont peut-être des eaux plus exposées à la prolifération de Legionella car ces bactéries nécessitent pour leur croissance la présence de soufre organique comme la cystéine. Il faut donc être particulièrement vigilant lors de l'exploitation de ce type d'eau,

- au plan de la flore autochtone : certaines eaux sulfurées se caractérisent par l'apparition à l'émergence, ou après l'émergence, de "bioglées " (appelées aussi "planctons thermaux ») en quantités plus ou moins importantes. Ces manifestations signalent une activité microbiologique intense et une flore particulière qui, s'il ne semble pas qu'elle ait une incidence au plan sanitaire, est considérée comme douée d'activité pharmacologique propre.

\section{CONCLUSIONS}

Les eaux sulfurées constituent une classe d'eaux minérales particulièrement intéressante mais qui reste encore mal connue. Un certain nombre de problèmes restent posés et il est possible de les regrouper sous forme de questions, en deux rubriques:

— au plan pratique :

- captage : comment capter une eau minérale sulfurée dont l'origine géologique n'est pas clairement définie, sans risquer de la dénaturer ou de lui faire perdre un caractère capital ?

- exploitation : comment concevoir les installations techniques pour préserver ou favoriser l'apparition de certaines espèces du soufre réduit?

- contrôle de la qualité : comment assurer la surveillance réglementaire des eaux minérales sulfurées et comment interpréter les variations inévitables de composition chimique entre émergence et points d'usage ?

- au plan fondamental :

- ne devrait-on pas reconsidérer la définition des eaux minérales sulfurées en intégrant un seuil de concentration en $\mathrm{S}(-2)$ en dessous duquel on ne pourrait pas faire état du caractère sulfuré ?

- de même, la classification ne devrait-elle pas être plus proche des réalités géologiques ou physico-chimiques ou pharmacologiques?

- l'intérêt de la recherche devrait être relancé afin de compléter utilement nos connaissances sur certaines espèces du soufre réduit comme les dérivés organiques, sur l'activité pharmacologique des eaux sulfurées après oxydation partielle et sur la flore microbienne intrinsèque de ce type d'eau minérale.

\section{Bibliographie}

[1] Anglada J. - Traité des Eaux Minérales et des Etablissements Thermaux du Département des Pyrénées Orientales. Paris : Baillère, Librairie, rue de l'Ecole de Médecine, 1833 , 2 volumes. 


\section{EAUX MINÉRALES SULFURÉES}

[2] Boulègue J. - Géochimie du soufre dans les milieux réducteurs. Thèse : Doctorat ès Sc. Physiques, Paris VII, 1978, 49.

[3] Boulègue J., Popoff G. - Nouvelles méthodes de détermination des principales espèces ioniques du soufre dans les eaux naturelles. Journal Français d'Hydrologie, 1979, 10, 2, 29. pp. 83-90.

[4] Bourrus C. - Les eaux sulfurées, leur application thérapeutique à travers une base de données bibliographiques informatisée. Thèse : Pharmacie : Bordeaux 2, 1992, 59.

[5] Canellas J., Toussaint C., Courtès C., Nguyen BA C. - Au sujet de l'interaction entre une eau chlorosulfurée sodique et l'acétylcholine. Journal Français d'Hydrologie, 1991, 22, 1, pp. 127-134.

[6] CAZAuX J. - Classification chimique des eaux minérales. In : Précis de Pharmacologie et de Thérapeutique hydrominérale des principales stations thermales françaises. Paris : 1'Expansion, 1964, pp. 21-30.

[7] Courtès C., Toussaint C., Nguyen BA C., Pujol J., CANEllas J. - Déterminations couplées des caractéristi- ques chimiques et des propriétés pharmacodynamiques d'une eau minérale sulfurée, In Journées de Pharmacie de Méditerranée Latine, Bordeaux, 26 septembre 1991.

[8] Fourcroy, Delaporte. - Analyse chimique de l'eau minérale sulfureuse d'Enghien, pour servir à l'histoire des eaux sulfureuses en général. Paris : Cuchet, 1778.

[9] LAUGIER R., MARTIN H. - Les eaux sulfurées sodiques: mythe ou réalité ? Presse Thermale et Climatique, 1988. 125, 4, pp. 191-192.

[10] Nguyen BA C., Ohayon-Courtès C., Toussaint C., Pu Jol J., Canellas J., Pouchan P. - Spécificités physicochimiques et pharmacodynamiques des eaux thermominérales dans leur environnement hydrogéologique, In Journées de Pharmacie de Méditérannée Latine, Grenade, Espagne, 2-5 octobre 1992.

[11] OHAYON-COURTÈs C. - L'évolution des espèces réduites du soufre dans les eaux minérales. Cas des eaux sulfurées sodiques. Thèse : Doctorat ès Sc. Pharmaceutiques: Bordeaux 2, 1992, 1 